\title{
Investigation of the Permeability of Soil-rock Mixtures Using Lattice Boltzmann Simulations
}

\author{
Lei Jin ${ }^{1 *}$, Yawu Zeng ${ }^{2}$, Jingjing $\mathrm{Li}^{1}$, Hanqing Sun ${ }^{2,3}$ \\ ${ }^{1}$ School of Civil Engineering, Hubei Polytechnic University, Huangshi, 435003, China \\ 2 School of Civil Engineering, Wuhan University, Wuhan, 430072, China \\ ${ }^{3}$ Department of Infrastructure Engineering, The University of Melbourne, Melbourne, Parkville, VIC 3010, Australia \\ *Corresponding author, e-mail: whujinlei@whu.edu.cn
}

Received: 21 April 2020, Accepted: 04 January 2021, Published online: 19 January 2021

\begin{abstract}
Based on the discrete element method and the proposed virtual slicing technique for three-dimensional discrete element model, random pore-structural models of soil-rock mixtures are constructed and voxelized. Then, the three-dimensional lattice Boltzmann method is introduced to simulate the seepage flow in soil-rock mixtures on the pore scale. Finally, the influences of rock content, rock size, rock shape and rock orientation on the simulated permeability of soil-rock mixtures are comprehensively investigated. The results show that the permeability of soil-rock mixtures remarkably decreases with the increase of rock content. When the other conditions remain unchanged, the permeability of soil-rock mixtures increases with the increase of rock size. The permeability of soil-rock mixtures with bar-shaped rocks is smaller than that of soil-rock mixtures with block-shaped rocks, but larger than that of soil-rock mixtures with slab-shaped rocks. The rock orientation has a certain influence on the permeability of SRMs, and the amount of variation changes with the rock shape: when the rocks are bar-shaped, the permeability is slightly decreased as the major axes of these rocks change from parallel to perpendicular with respect to the direction of main flow; when the rocks are slab-shaped, the permeability decreases more significantly as the slab planes of these rocks change from parallel to perpendicular with respect to the direction of main flow.
\end{abstract}

Keywords

soil-rock mixtures, discrete element method, lattice Boltzmann method, permeability

\section{Introduction}

Soil-rock mixtures (SRMs, for short) formed since the Quaternary Period are a type of extremely inhomogeneous geomaterial composed of coarse rock fragments, fine grained soil with pores [1, 2]. SRMs are commonly found in most geological bodies such as slopes, fault zones and dam foundations [3, 4]. When analyzing engineering problems such as the stability of slopes undergoing rainfall infiltration, water inrush of fault zones during tunnel excavation and leakage in dam foundations, the permeability of SRMs needs to be determined beforehand. Therefore, it is of great significance to gain insight into the permeability of SRMs.

In recent decades, some scholars have studied the permeability of SRMs through in-situ tests, laboratory tests and numerical simulations. Gao et al. [5] measured the permeability coefficient of SRMs through in-situ water injection tests. Zhou et al. [6] and Wang et al. [7] analyzed the influences of such factors as rock content and confining pressure on the permeability coefficient of SRMs through laboratory tests.

Some of the limitations confronting physical tests, such as time-consuming, limited sample size and difficulty in directly observing the internal seepage field, can be effectively released by numerical simulations. As the most popular numerical simulation method in geotechnical engineering, the finite element method has been used in several studies to investigate the permeability of SRMs [8,9]. However, the complex internal structure of SRM makes it time-consuming and difficult to generate high-quality finite element meshes. To this end, Chen et al. [10] introduced the numerical manifold method based on regular grids to determine the permeability coefficient of SRMs.

The numerical simulations of seepage in SRMs need further improvement in several aspects. First, the known 
numerical models are mainly developed for the continuum, while SRMs are typically composed of discrete granular materials. Second, the previous numerical simulations are two-dimensional, but seepage occurs in the three-dimensional pore space.

Compared with the traditional numerical simulation methods, the lattice Boltzmann method (LBM) is a mesoscopic simulation method, which improves the insufficient calculation accuracy caused by the assumption of macro-continuity and has no limitations of the micro-molecular dynamic models in the time and space. The LBM has many advantages including clear definitions of physical conditions, simple implementation of algorithms, convenient boundary processing, and easy parallelism [11]. Pore-scale flow simulations based on the LBM and the pore-structural model of porous medium have been developed to study the micro-mechanism of macroscopic percolation [12-21]. However, to our knowledge, three-dimensional LBM based simulation of the seepage in SRMs has not been reported yet.

The LBM-based simulation of seepage requires the construction of pore-structural models of the SRMs. Structure models of SRMs are generally constructed with two classes of methods: the measured structure modeling methods based on digital image processing $[1,9]$ and the random structure modeling methods $[4,8,10]$. The former can more realistically describe the meso-structural characteristics of the physical models of SRMs, but is difficult in independently changing a specified structural parameter, while the latter can quickly generate a specific structure model as required. Among the existing random structure modeling methods, the granular discrete element method (DEM) can describe the components with the heterogeneous and discontinuous characteristics in SRMs. The DEM modeling method has been widely used to study the strength and deformation characteristics of SRMs [22, 23] and to simulate the seepage of porous media in combination with the LBM $[15,19]$.

In this study, three-dimensional random pore-structural models are constructed based on the DEM for the SRMs with different rock contents, rock sizes, rock shapes, and rock orientations. Then, these pore-structural models of SRMs are discretized with the proposed virtual slicing technique. Finally, the pore-scale seepage in these SRMs is simulated based on the three-dimensional LBM, and the influences of various factors are analyzed on the permeability of the SRMs.

\section{Three-dimensional discrete element models of SRMs}

\subsection{A brief introduction to DEM}

In this study, the pore-structural models of SRMs are generated with the DEM [24], which can model the movement and interaction of assemblies composed of rigid spherical particles. The calculations performed in the DEM alternate between the application of Newton's second law to the particles and a force-displacement law at the contacts. The Newton's second law is used to determine the motion of each particle arising from the contact and body forces acting upon it. The force-displacement law is used to update the contact forces arising from the relative motion at each contact. The commonly used force-displacement law is the spring-viscous damping model of linear contact, and the shear contact force also observes the law of friction.

In the DEM, a common way to make an irregular shape is by using the clump logic. A clump is composed of several spherical particles with their relative positions kept fixed and the contact calculation between them is skipped during calculation cycles, which can save computing time greatly [25].

\subsection{Schemes of DEM modeling for SRMs}

As described above, SRMs consist of coarse-grained rocks scattered in fine-grained soil matrix. It should be noted that the called "rock" and "soil" in SRMs both are relative to the studying scale. That is, the "soil" in SRMs is only one relative conception, which is different from the granular soil, such as "silty soil", "clay" and so on, in the general conception. The size range of the "soil" changes accordingly with the varying of the studying scale, and the upper limits may range from a few millimeters to a few centimeters, or even tens of centimeters. Xu and Medley [1, 2] found that SRMs are scale-independent and suggested that the soil/rock threshold of SRMs can be expressed as

$d_{\mathrm{S} / \mathrm{RT}}=0.05 L_{\mathrm{C}}$,

where $L_{C}$ is the engineering characteristic size of the problem studied, for example, it is the slope height for slope engineering and the sample diameter for the indoor mechanical tests.

Following the laboratory seepage experiments, the boundary wall in the DEM model is set to be a cylinder with a diameter of $0.3 \mathrm{~m}$ and a height of $0.9 \mathrm{~m}$. Thus, the soil/rock threshold of SRMs can be calculated as $0.015 \mathrm{~m}$. To save the computational time of the subsequent LB simulations, the soil particles are simplified as spheres with diameters of $0.015 \mathrm{~m}$. 
The major objective of this paper is to study the influence of rock content, rock size, rock shape and rock orientation on the permeability of SRMs. The specific definitions and ranges of these parameters are considered in the following modeling schemes.

(1) The rock contents $\left(C_{\mathrm{R}}\right)$ are $20 \%, 30 \%$ and $40 \%$. Here, the rock content is defined as the ratio of the mass of all rock particles to the total mass of the SRM sample.

(2) The rock shapes are block-shaped, bar-shaped and slab-shaped, as shown in Fig. 1. The rock shape is characterized by the ratio of the three axial lengths of the rock: a block-shaped rock is represented by 1 sphere with the ratio of $1: 1: 1$; a bar-shaped rock is represented by a clump with the ratio of $1: 1: 3$, which is composed of 3 spheres; a slab-shaped rock is represented by a clump with the ratio of $1: 3: 3$, which is composed of 13 spheres.

(3) The rock sizes $(D)$ are $0.03 \mathrm{~m}, 0.04 \mathrm{~m}$, and $0.05 \mathrm{~m}$. The sizes of a bar-shaped and slab-shaped rock both refer to the diameters of the equivalent spheres with the same volumes.

(4) The rock orientations $(\theta)$ are $0^{\circ}$, random and $90^{\circ}$. The orientation of a bar-shaped rock refers to the angle between the major axis of this rock and the main direction of flow. The orientation of a slab-shaped rock refers to the angle between the slab plane (perpendicular to the minor axis) of this rock and the main direction of flow. " $\theta=$ random" denotes that the rock orientations are random values.

Additionally, when studying the influence of such factors as rock content on the permeability of SRMs, the compactness of samples should be controlled and remain unchanged. The general practice of keeping the density of soil matrix in the SRM samples to be constant $[7,8,10]$ is also followed in this study, which is implemented by setting the porosity of soil matrix to be 0.40 .

\subsection{Methods of DEM modeling for SRMs}

A discrete element model can be constructed with three kinds of methods: accumulation under gravity; boundary compression method; and radius expansion method. It is believed that the particle packing obtained by the radius expansion method is more uniform [24, 25]. Hence, the

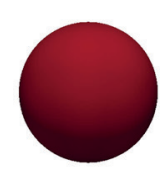

Block-shaped

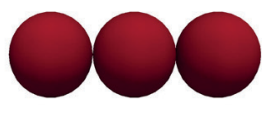

Bar-shaped

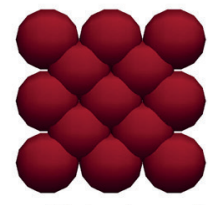

Slab-shaped
Fig. 1 schematic diagram of the rock shapes of SRMs radius expansion method is used to construct the threedimensional discrete element models of SRMs in this study. The main steps are as follows:

(1) Set the seed of random number generator, generate the boundary walls according to the selected size and shape of the sample.

(2) Generate balls (their radii are appropriately reduced as required) for the rock particles according to the selected rock size and rock content. The balls are randomly distributed in the sample region, and the radii of these balls are then expanded back to the required rock size.

(3) Replace the balls generated in step (2) according to the selected rock shape and rock orientation. The three principles of "volume equivalent", "volume center equivalent" and "mass equivalent" are followed in the replacement.

(4) Generate balls (their radii are appropriately reduced as required) for the soil particles in the remaining region of the sample, and then the radii of these soil balls are expanded to the required size of soil particles.

(5) Set meso-mechanical parameters for the rock and soil particles, perform DEM calculation to bring the sample to equilibrium. During DEM calculation, the velocities of particles need to be reset to zero every certain number of cycles to prevent particles from escaping the boundary due to possibly excessive contact force.

In our work, the density of $2650 \mathrm{~kg} / \mathrm{m}^{3}$, the normal and shear stiffness of $1.0 \times 10^{7} \mathrm{~N} / \mathrm{m}$ and the friction coefficient of 0.5 are set for the solid particles in the DEM modeling of SRMs. It should be noted that the orientation of some rock particles generated in step (3) may change slightly during the DEM calculation in the step (5), but it has little effect on the overall rock orientation of SRM samples. By changing the random number seed in step (1), 3 parallel samples can be prepared for each modeling scheme. Considering the computational efficiency in the subsequent lattice Boltzmann simulations, the middle part ranging $0.3-0.6 \mathrm{~m}$ from the bottom surface in each discrete element model is selected, as shown in Fig. 2.

\section{LB simulations of the permeability}

\subsection{MRT-LBE model}

The LBM treats the fluid as an assembly of mesoscopic particles. The fluid domain is discretized into cubic lattices with side $h$. Fluid particles at each node would collide and then be allowed to move to its immediate neighbors with different velocities $\boldsymbol{e}_{i}$. The interaction and propagation of those particles are governed by the following lattice Boltzmann equation (LBE) (Eq. (2)) [26]: 

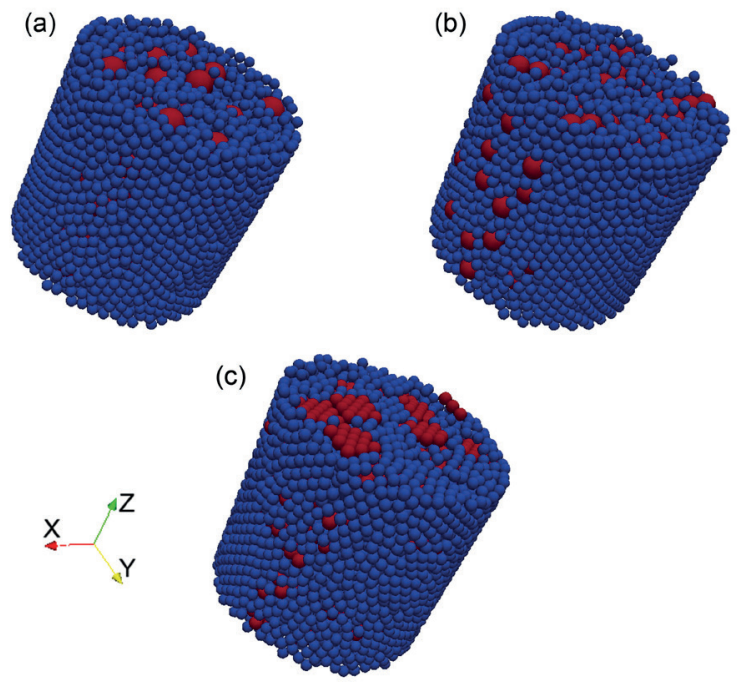

Soil particles

Rock particles

Fig. 2 Three-dimensional discrete element models of SRMs with rock contents of $30 \%$ and rock sizes of $0.04 \mathrm{~m}$; (a) Block-shaped rocks, (b)

Bar-shaped rocks and $\theta=90^{\circ}$, (c) Slab-shaped rocks and $\theta=90^{\circ}$

$f_{i}\left(\boldsymbol{x}+\boldsymbol{e}_{i} \Delta t, t+\Delta t\right)-f_{i}(\boldsymbol{x}, t)=\Omega_{i}$,

where $f_{i}(\boldsymbol{x}, t)$ is the fluid density distribution function at that site $\boldsymbol{x}$ at that moment $t$ in the direction of $i$, and $\Delta t$ and $\Omega_{i}$ are the time-step increment and the collision operator.

The Bhatnagar-Gross-Krook (BGK) single-relaxationtime (SRT) collision operator [26] is the most adopted. However, several problems are encountered with this popular method such as numerical instability and viscosity dependence of boundary locations. Particularly, the viscosity dependent boundary conditions pose a severe problem for simulating flow through porous media because the permeability becomes viscosity dependent, while it should be a characteristic of the physical properties of porous medium alone. The deficiencies inherent in the BGK model can be significantly reduced by using a multiple-relaxation-time (MRT) approach [27, 28], which separates the relaxation times for different kinetic modes and allows tuning to improve numerical stability and accuracy.

For clarity, the evolution equation of the MRT-LBE is divided into two steps, namely collision and streaming, respectively expressed as:

$$
\begin{aligned}
& f_{i}{ }^{\prime}(\boldsymbol{x}, t)=f_{i}(\boldsymbol{x}, t)-\Lambda_{i j}\left[f_{j}-f_{j}^{(\mathrm{eq})}\right], \\
& f_{i}\left(\boldsymbol{x}+\boldsymbol{e}_{i} \Delta t, t+\Delta t\right)=f_{i}{ }^{\prime}(\boldsymbol{x}, t),
\end{aligned}
$$

where $-\Lambda$ is the collision matrix and $f_{j}^{(\mathrm{eq})}$ is the equilibrium distribution function. The collision step involves only local calculations and can be written in vector form as
$\boldsymbol{f}^{\prime}=\boldsymbol{f}-\Lambda\left[\boldsymbol{f}-\boldsymbol{f}^{(\mathrm{eq})}\right]$

The transformation matrix $\boldsymbol{M}$ relates the distribution functions represented by $\boldsymbol{f}$ to their moments represented by $\boldsymbol{m}$, as in the following:

$\boldsymbol{m}=\boldsymbol{M} \cdot \boldsymbol{f}, \boldsymbol{f}=\boldsymbol{M}^{-1} \cdot \boldsymbol{m}$.

Multiplied by the transformation matrix $\boldsymbol{M}$, the collision step in Eq. (5) can be executed in the moment space as the following:

$\boldsymbol{m}^{\prime}=\boldsymbol{m}-\boldsymbol{S}\left[\boldsymbol{m}-\boldsymbol{m}^{(\mathrm{eq})}\right]$,

where $\boldsymbol{m}^{(\mathrm{eq})}=\boldsymbol{M} \cdot \boldsymbol{f}^{(\mathrm{eq})}$ is the equilibrium function in the moment space and $\boldsymbol{S}=\boldsymbol{M} \Lambda \boldsymbol{M}^{-1}$ is the diagonal relaxation matrix.

After the collision step is performed, the post-collision moment $\boldsymbol{m}^{\prime}$ is inversely converted to the post-collision distribution function $\boldsymbol{f}^{\prime}$ in the velocity space, and then the standard streaming step in Eq. (4) can be executed.

The popular D3Q19 model using a cubic lattice with 19 velocities is adopted and the corresponding transformation matrix $\boldsymbol{M}$ is given in [27]. For the D3Q19 model, the 19 moments are

$$
\begin{gathered}
\boldsymbol{m}=\left(\rho, e, \varepsilon, j_{x}, q_{x}, j_{y}, q_{y}, j_{z}, q_{z}, 3 p_{x x}, 3 \pi_{x x},\right. \\
\left.p_{w w}, \pi_{w w}, p_{x y}, p_{y z}, p_{x z}, m_{x}, m_{y}, m_{z}\right)^{\mathrm{T}},
\end{gathered}
$$

and the equilibria $\boldsymbol{m}^{(\mathrm{eq})}$ can be defined as:

$$
\begin{aligned}
\boldsymbol{m}^{(\mathrm{eq})}= & \rho\left(1,-11+19 u^{2}, \alpha+\beta u^{2}, u_{x},-\frac{2}{3} u_{x},\right. \\
& u_{y},-\frac{2}{3} u_{y}, u_{z},-\frac{2}{3} u_{z}, 3 u_{x}^{2}-u^{2}, u_{y}^{2}-u_{z}^{2}, \\
& \left.u_{x} u_{y}, u_{y} u_{z}, u_{x} u_{z}, \frac{\gamma p_{x x}^{(\mathrm{eq})}}{\rho}, \frac{\gamma p_{w w}^{(\mathrm{eq})}}{\rho}, 0,0,0\right)^{\mathrm{T}},
\end{aligned}
$$

where $\alpha, \beta$ and $\gamma$ are free parameters.

Corresponding the particular order of moments used here, the diagonal relaxation matrix $S$ is given by

$$
\begin{array}{r}
\boldsymbol{S}=\operatorname{diag}\left(0, s_{e}, s_{\varepsilon}, 0, s_{q}, 0, s_{q}, 0, s_{q}, s_{v},\right. \\
\left.s_{\pi}, s_{v}, s_{\pi}, s_{v}, s_{v}, s_{v}, s_{m}, s_{m}, s_{m}\right) .
\end{array}
$$

The macroscopic fluid variables, density $\rho$ and velocity $\boldsymbol{u}$, can be recovered from the moments of the distribution functions as follows:

$\rho=\sum_{i=0}^{18} f_{i}, \mathbf{u}=\frac{1}{\rho} \sum_{i=0}^{18} f_{i} \mathbf{e}_{i}$,

while the fluid pressure field $p$ is determined by the following equation of state (Eq. (12)): 
$p=\rho C^{2} / 3$,

where $C=h / \Delta t$ is the lattice speed.

The kinematic viscosity $v$ of the fluid is, however, not directly used in the LB model but implicitly determined by the discretization and numerical parameters as

$v=\frac{1}{3}\left(\frac{1}{s_{v}}-\frac{1}{2}\right) \frac{h^{2}}{\Delta t}$

To simulate the Darcy's flow through porous media, Pan and coauthors [28] suggested that the linear MRT-LBE scheme can be used, in which all the nonlinear velocity terms are omitted in the equilibria given by Eq. (9), i.e.,

$$
\begin{gathered}
\boldsymbol{m}^{\text {(eq) }}=\rho\left(1,-11,3, u_{x},-\frac{2}{3} u_{x}, u_{y},-\frac{2}{3} u_{y}, u_{z},\right. \\
\left.-\frac{2}{3} u_{z}, 0,0,0,0,0,0,0,0,0,0\right)^{\mathrm{T}} .
\end{gathered}
$$

They also concluded that the relaxation parameters following the two-relaxation-time (TRT) model is with good effect, i.e.,

$s_{e}=s_{\varepsilon}=s_{\pi}=s_{v}=\frac{1}{\tau}, s_{m}=s_{q}=8 \frac{\left(2-s_{v}\right)}{\left(8-s_{v}\right)}$.

Hereafter, the linear MRT-LBE and the relaxation parameters given in Eq. (15) were used unless otherwise stated.

\subsection{Voxelization of discrete element models}

The discrete element models constructed for the LB simulation of the seepage in SRMs should be adapted to regular cubic lattices beforehand, and this process is called "voxelization", which is implemented with digital image processing technique in this study. The key problem is how to obtain a series of sliced images of a three-dimensional discrete element model of SRM. Fortunately, the virtual slicing technique [29] developed for three-dimensional discrete element models can solve this problem. The basic principles and implementation method of this technique are briefly introduced in this subsection.

It is noted that the three-dimensional discrete element model of SRM is a packing of sphere particles and the overlapping of a sphere and a certain cutting plane is a circle. In a three-dimensional Cartesian coordinate system, if a cutting plane parallel to the $X O Y$ coordinate plane with a $Z$ coordinate of $z_{\mathrm{cp}}$ overlaps a sphere with center at $\left(x_{0}, y_{0}, z_{0}\right)$ and radius of $R$, the overlapping circle is expected to be located on the cutting plane, whose center is at $\left(x_{0}, y_{0}, z_{\mathrm{cp}}\right)$ and the radius $R^{*}$ is calculated by

$$
R^{*}=\sqrt{R^{2}-\left(\mathrm{z}_{\mathrm{cp}}-z_{0}\right)^{2}} .
$$

In this way, all circles can be obtained, which result from the overlapping of the packing spheres and the specified cutting plane.

The above basic principles can be implemented on the MATLAB platform to obtain a series of slices of the threedimensional discrete element model of SRM. The main steps include:

(1) Generate three-dimensional discrete element model of SRM;

(2) Search for the spheres overlapping with a specified cutting plane, calculate the coordinates of the centers and radii of all overlapping circles, and export these data;

(3) For each slice in the batch mode, import the data regarding the centers and radii of overlapping circles into MATLAB, draw all the overlapping circles, and fill them with the black color (the background color is white) representing the solid region.

In this study, the middle part ranging $0.3-0.6 \mathrm{~m}$ from the bottom surface in each discrete element model is sliced along the axial direction ( $\mathrm{Z}$ axis). For example, Fig. 3 shows the generation process of the slice image at the middle of the model in Fig. 2(c). Considering that LBM calculation domain is generally a cuboid, the solid area on each slice image also includes the region between the outer circular boundary and the circumscribed square, as shown in Fig. 3(c). When the discrete element model is sliced at $0.001 \mathrm{~m}$ intervals and the pixel size of each slice image is also set to be $0.001 \mathrm{~m}$ (i.e., $300 \times 300$ pixels), a discretized model consisting of 27,000,000 cubic lattices can be generated (i.e., $h=0.001 \mathrm{~m}$ ).

\subsection{Definition of boundary conditions and acceleration of computation}

Appropriate boundary conditions should be defined for the LB simulation of seepage flow. In this paper, the flow is driven by the pressure difference between the inlet and outlet along the positive direction of $Z$ axis, and the

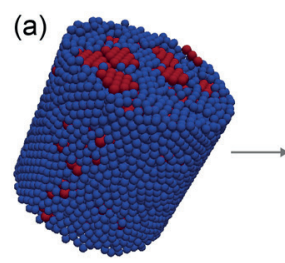

(b)

(c)

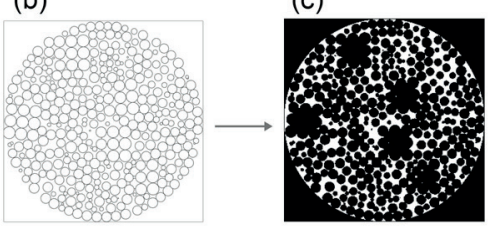

Fig. 3 Slicing of discrete element model; (a) Discrete element model of SRM $\left(C_{\mathrm{R}}=30 \%, D=0.04 \mathrm{~m}\right.$, slab-shaped and $\left.\theta=90^{\circ}\right)$, (b) Overlapping circles on the middle slice, (c) Filing for the solid region 
associated pressure boundary is treated by the Zou/He scheme [30]. The bounce-back rule is used at the surrounding walls and internal solid surfaces where the momenta of the fluid particles should be reversed. This method is attractive for its simplicity and computational efficiency in imposing no-flow conditions on irregularly shaped walls. Before performing the bounce-back scheme, it is necessary to separate the lattices into the solid lattices and the fluid lattices with the following method: the voxel with pixel value of 0 (black) belongs to the solid lattices, while the voxel with pixel value of 255 (white) belongs to the fluid lattices.

The LBM-based pore-scale seepage simulation is likely to be computationally demanding especially for three-dimensional cases with large domain sizes, so the distributed computing platform PALABOS [31] is used with 8 computer threads for parallel computing. In addition, the solid lattices are further distinguished into the boundary solid lattices and the interior solid lattices in the following way: if the 26 lattices adjacent to a solid lattice shown in Fig. 4 are all solid lattices, then the solid lattice is marked as an interior solid lattice; otherwise, it is marked as a boundary solid lattice. During the process of LBM computing, the bounce-back rule is implemented for the boundary solid lattices, but skipped for the interior solid lattices. Fig. 5 shows the three types of lattices marked for the model in Fig. 2(c). It can be seen that a relatively large region of the total domain is occupied by the solid lattices (marked as red), so the computing time will be effectively saved.

\subsection{Permeability and simulation parameters}

The capability of a porous medium to allow the passage of fluid is generally characterized by the intrinsic permeability or permeability coefficient. The intrinsic permeability is controlled by the properties of the porous medium (such as the porosity and specific surface), while

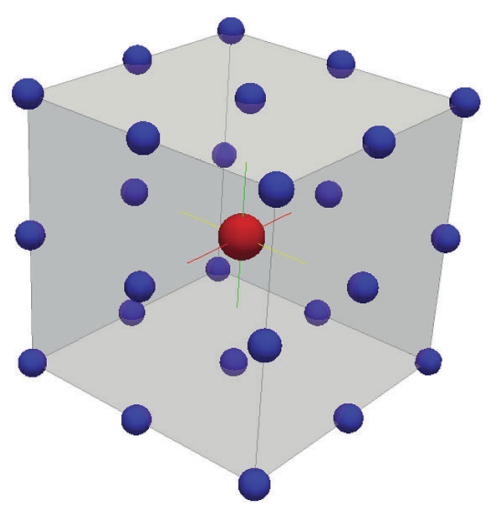

Fig. 4 The 26 lattices adjacent to a solid lattice the permeability coefficient is related not only to the properties of the porous medium but also to the properties of the fluid (such as the density and viscosity). In this paper, it is the intrinsic permeability of SRMs that is investigated, which is hereinafter termed as "permeability" for convenience.

Previous experimental and theoretical studies have shown that the main factors affecting the permeability of granular materials are porosity, specific surface and tortuosity of flow paths. It is generally recognized that the permeability increases with the porosity and decreases with the specific surface and tortuosity [32,33].

Natural SRMs are extremely heterogeneous porous medium, in which over-sized rock fragments often exist. The major objective of this article is to explore the influence of rock fragments on the permeability of SRMs. Here, the permeability $(k)$ is calculated from the simulated seepage field through the Darcy's law formulated as

$$
k=\frac{\mu \bar{u}}{\nabla p}=\frac{\rho v \overline{u l}}{p_{\text {in }}-p_{\text {out }}},
$$

where $\mu$ is the dynamic viscosity of the fluid; $\bar{u}$ represents the average flow velocity; $\nabla p$ is the pressure gradient; $l$ is the length of the model along the direction of main flow; $p_{\text {in }}$ and $p_{\text {out }}$ are the pressure at inlet and outlet, respectively.

The seepage fluid in SRMs is usually water, but LB simulation of such fluid flow with a small viscosity presents a challenge. Specifically, in this case either lattice size $h$ needs to be very small or relaxation time $\tau$ has to be very close to 0.5 . The former option may give rise to a prohibitively large-scale model, while the latter may suffer from numerical instability [34]. In view of the above dilemma and considering that the permeability of a porous media is only related to its own properties, the simulated seepage fluid in this paper is assumed to have a viscosity of

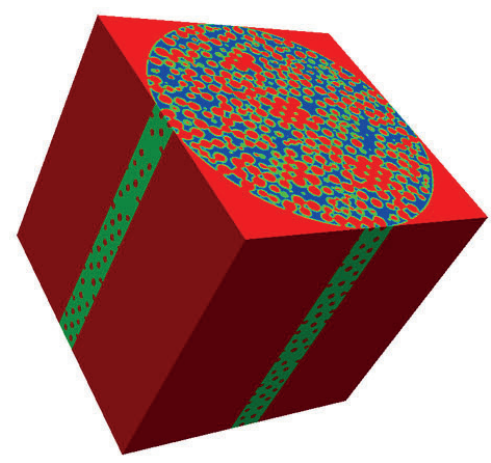

Fig. 5 The three types of lattices marked for the model in Fig. 2(c) (blue region represents the fluid lattices, green region represents the boundary solid lattices, and red region represents the interior solid lattices) 
$1 \times 10^{-2} \mathrm{~m}^{2} / \mathrm{s}$ and a density of $1000 \mathrm{~kg} / \mathrm{m}^{3}$. The value of $\tau$ is 0.8 and each relaxation parameter in the MRT-LBE can be determined by Eq. (15).

The resolution of the slice images or the number of voxels has an important influence on the calculation accuracy of LBM [17]. The study of Pan et al. [13] suggested that when the diameter of the packing sphere is over 12 times of the lattice size, the calculated permeability would not change with the resolution. In consideration of the minimum solid particle diameter of $0.015 \mathrm{~m}$, the lattice size $h$ is $0.001 \mathrm{~m}$ in this paper.

The Darcy's law requires that the flow is laminar, and when the derived permeability is constant, the flow is considered to be laminar. Hence some LB simulations should be run on a test model to determine the adequate pressure difference to be applied across the medium in the direction of flow.

The LB simulations end only when the flow has reached a steady state, which is defined as the flow field with the standard deviation of the kinetic energy over the entire domain measured over 1,000 time steps falling below $0.01 \%$ of the mean kinetic energy over the entire domain averaged over the same time interval. After then, the permeability can be calculated from the Darcy's law based on the defined pressure gradient, the calculated average fluid velocity per unit cross-sectional area normal to the flow, and the fluid viscosity.

\subsection{Validation of LB simulation}

Before applying the LBM to simulate the seepage of SRMs, we tested the accuracy of LB simulation by calculating the laminar flow of viscous fluid in an infinite straight circular pipe. The analytical solution can be derived based on the Poiseuille's law:

$u=\frac{p_{\text {in }}-p_{\text {out }}}{4 \mu l}\left(r_{0}^{2}-r^{2}\right)$,

$\bar{u}=\frac{p_{\text {in }}-p_{\text {out }}}{8 \mu l} r_{0}^{2}$,

where $r_{0}$ is the radius of the pipe and $r$ is the distance from the calculating point to the central axis of the pipe.

To be consistent with aforementioned SRMs samples, the computational domain of the simulated flow in a straight circular pipe is also a cubic region with a side length of $0.3 \mathrm{~m}$, as shown in Fig. 6. In order to simulate infinite length, periodic boundary is set at the inlet and outlet, where the pressure difference of $0.3 \mathrm{~Pa}$ is maintained to satisfy the assumption of laminar flow.
Comparison is made in Fig. 7 between the result of LB simulation and the analytical solution for the flow velocity on the middle cross-section, and it can be seen that the two solutions are in good agreement. The average velocity calculated by the Poiseuille's law is 0.000281 and that simulated by LBM is 0.000284 , with a relative error of $1.07 \%$. The deviation is likely to be caused mainly by the fact that the pipe surface is not ideally smooth after voxelization of the LB model.

\subsection{Permeability simulation}

In this section, seepage flow in porous medium composed of mono-sized spherical solid particles is simulated with the LBM. The porosity is 0.4 and particle size is respectively $0.015,0.02,0.03,0.04$ and $0.05 \mathrm{~m}$, which are all within the particle size range of SRMs as described in Section 2.2.

The seepage flow in porous media with the particle size of $0.05 \mathrm{~m}$ is simulated to determine the adequate pressure difference. When the pressure difference is respectively set as $0.1,1,10$ and $100 \mathrm{kPa}$, the correspondingly simulated permeability is $1.547 \times 10^{-6} \mathrm{~m}^{2}, 1.548 \times 10^{-6} \mathrm{~m}^{2}$,

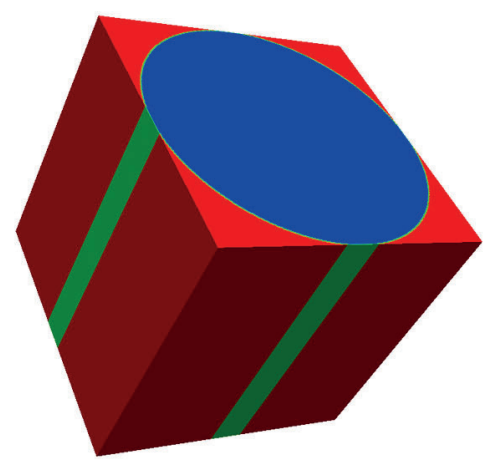

Fig. 6 Lattices of the model of flow in a straight circular pipe

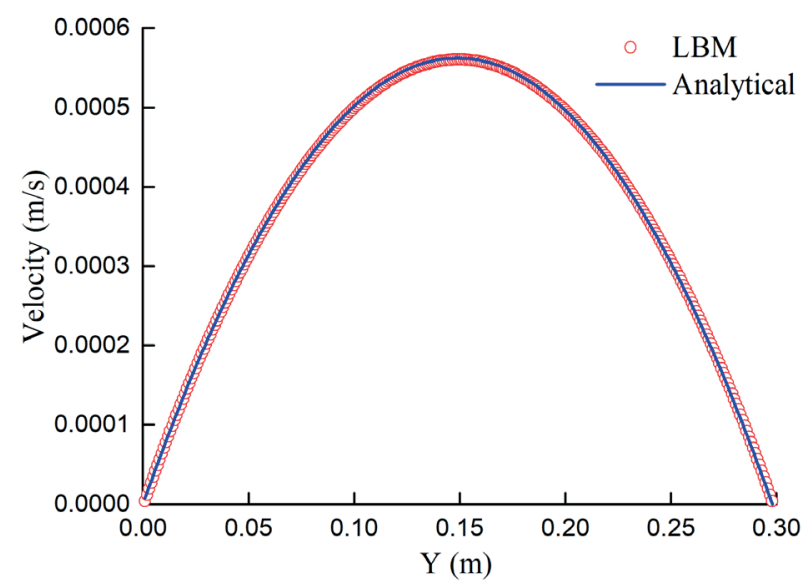

Fig. 7 Comparison between the result of LB simulation and the analytical solution for the flow velocity on the middle cross-section of the flow in the straight circular pipe 
$1.550 \times 10^{-6} \mathrm{~m}^{2}, 1.572 \times 10^{-6} \mathrm{~m}^{2}$. Hence, the pressure difference of $1 \mathrm{kPa}$ can be adopted to satisfy the assumption of Darcy's flow.

For the porous media with the particle size of $0.015 \mathrm{~m}$, when the lattice size $h$ is respectively set as 0.0006 , $0.00075,0.001,0.0015$ and $0.003 \mathrm{~m}$, the correspondingly simulated permeability is $1.210 \times 10^{-7} \mathrm{~m}^{2}, 1.210 \times 10^{-7} \mathrm{~m}^{2}$, $1.201 \times 10^{-7} \mathrm{~m}^{2}, 1.178 \times 10^{-7} \mathrm{~m}^{2}, 1.124 \times 10^{-7} \mathrm{~m}^{2}$. The test results indicate that the resolution of $h=0.001 \mathrm{~m}$ selected in this paper is adequate.

Previous studies have shown that when the porosity is the same, the permeability of porous medium with uniform particle size is approximately proportional to the square of the particle size, which is also described by the Hazen equation. For the porous medium composed of mono-sized spherical particles of $0.015,0.02,0.03,0.04$ and $0.05 \mathrm{~m}$, the linear regression of the simulated permeability and square of particle size is shown in Fig. 8. As can be seen, the linear correlation is well reproduced and the error is not more than $1 \%$, which further indicates that the LB simulation can faithfully describe the flow process in porous medium.

\section{Results and discussions}

Based on the modeling of pore structure with the above-mentioned discrete element method and the LB simulation of seepage, the permeability can be obtained of SRMs with different rock contents, rock sizes, rock shapes,

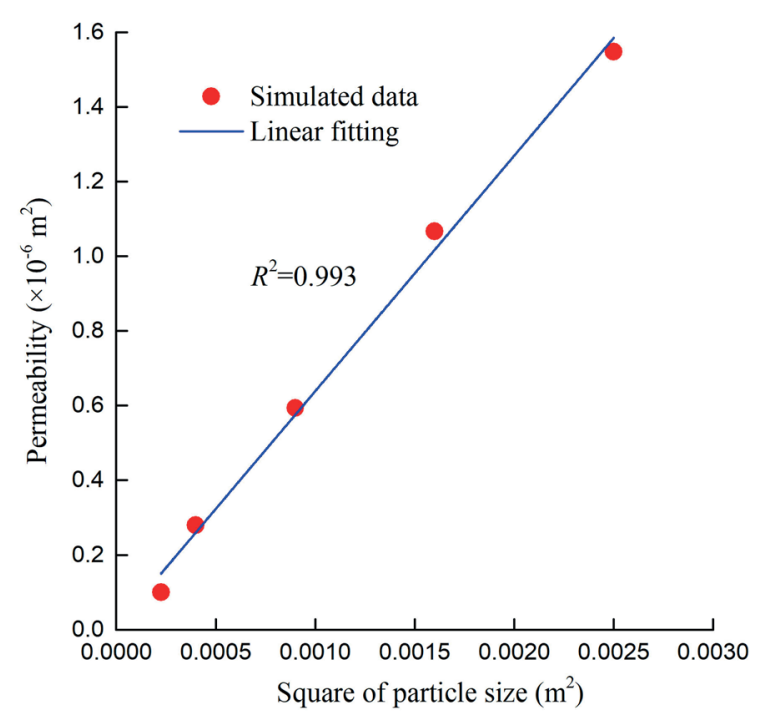

Fig. 8 Relation between permeability and square of particle size of porous medium

and rock orientations. The average is taken of the permeability for 3 parallel samples in each case. Tables 1-3 list this simulated permeability of every SRM model.

In addition to permeability, the LB simulation can also generate the pore-scale seepage field, as shown in Fig. 9. Fig. 9(a) shows the velocity contour plot of SRM $\left(C_{\mathrm{R}}=\right.$ $20 \%, D=0.05 \mathrm{~m}$ and block-shaped) with a permeability of $9.319 \times 10^{-8} \mathrm{~m}^{2}$, which is the largest of all models. Fig. 9(b) shows the velocity contour plot of SRM $\left(C_{\mathrm{R}}=40 \%\right.$, $D=0.03 \mathrm{~m}$, slab-shaped and $\theta=90^{\circ}$ ) with a permeability of $2.900 \times 10^{-8} \mathrm{~m}^{2}$, which is the smallest of all models.

Table 1 Simulated permeability of SRMs $\left(\times 10^{-8} \mathrm{~m}^{2}\right)(D=0.03 \mathrm{~m})$

\begin{tabular}{|c|c|c|c|c|c|c|c|}
\hline \multirow{2}{*}{$C_{\mathrm{R}}(\%)$} & \multirow{2}{*}{ Block-shaped } & \multicolumn{3}{|c|}{ Bar-shaped } & \multicolumn{3}{|c|}{ Slab-shaped } \\
\hline & & $\theta=0^{\circ}$ & $\theta=$ random & $\theta=90^{\circ}$ & $\theta=0^{\circ}$ & $\theta=$ random & $\theta=90^{\circ}$ \\
\hline 20 & 8.659 & 8.344 & 8.343 & 8.279 & 7.925 & 7.060 & 6.599 \\
\hline 30 & 6.658 & 6.293 & 6.135 & 6.108 & 6.055 & 5.138 & 4.462 \\
\hline 40 & 5.061 & 4.893 & 4.834 & 4.812 & 4.728 & 3.610 & 2.900 \\
\hline
\end{tabular}

Table 2 Simulated permeability of SRMs $\left(\times 10^{-8} \mathrm{~m}^{2}\right)(D=0.04 \mathrm{~m})$

\begin{tabular}{lcccccc}
\hline$C_{\mathrm{R}}(\%)$ & Block-shaped & $\theta=0^{\circ}$ & $\begin{array}{c}\text { Bar-shaped } \\
\theta=\text { random }\end{array}$ & $\theta=90^{\circ}$ & \multicolumn{2}{c}{$\begin{array}{c}\text { Slab-shaped } \\
\theta=0^{\circ}\end{array}$} \\
\hline 20 & 8.968 & 8.839 & 8.618 & 8.530 & 8.417 & 7.816 \\
30 & 7.404 & 7.123 & 7.073 & 7.041 & 6.668 & 5.191 \\
40 & 5.889 & 5.706 & 5.446 & 5.392 & 5.027 & 4.312 \\
\hline
\end{tabular}

Table 3 Simulated permeability of SRMs $\left(\times 10^{-8} \mathrm{~m}^{2}\right)(D=0.05 \mathrm{~m})$

\begin{tabular}{|c|c|c|c|c|c|c|c|}
\hline \multirow{2}{*}{$C_{\mathrm{R}}(\%)$} & \multirow{2}{*}{ Block-shaped } & \multicolumn{3}{|c|}{ Bar-shaped } & \multicolumn{3}{|c|}{ Slab-shaped } \\
\hline & & $\theta=0^{\circ}$ & $\theta=$ random & $\theta=90^{\circ}$ & $\theta=0^{\circ}$ & $\theta=$ random & $\theta=90^{\circ}$ \\
\hline 20 & 9.319 & 9.138 & 9.017 & 8.971 & 8.762 & 8.200 & 7.955 \\
\hline 30 & 7.692 & 7.542 & 7.442 & 7.364 & 7.117 & 6.403 & 6.040 \\
\hline 40 & 6.197 & 6.462 & 5.802 & 5.796 & 5.509 & 4.639 & 4.278 \\
\hline
\end{tabular}


As can be seen, with the decrease of simulated permeability, compared with Fig. 9(a), remarkably fewer flow channels appear in Fig. 9(b) and the flow velocity is smaller in the channels.

\subsection{Influence of rock content and rock size}

Figs. 10-12 show the influence of rock content and rock size on the permeability of SRMs. It can be seen that the permeability significantly decreases with the increase of rock content and increases with the increase of rock size.

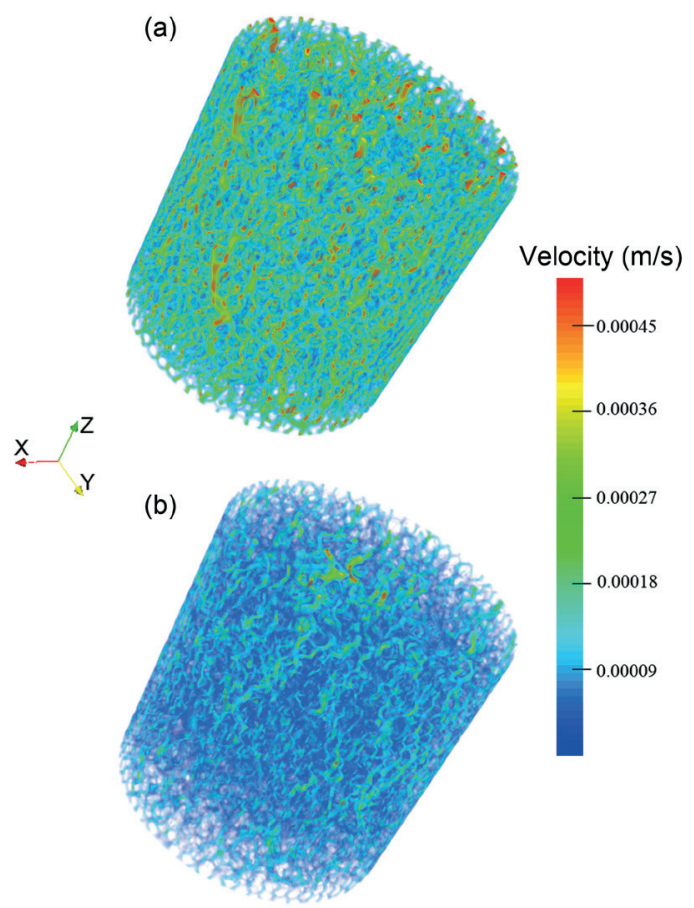

Fig. 9 Velocity contour plots of SRMs. (a) $C_{\mathrm{R}}=20 \%, D=0.05 \mathrm{~m}$ and block-shaped, (b) $C_{\mathrm{R}}=40 \%, D=0.03 \mathrm{~m}$, slab-shaped and $\theta=90^{\circ}$

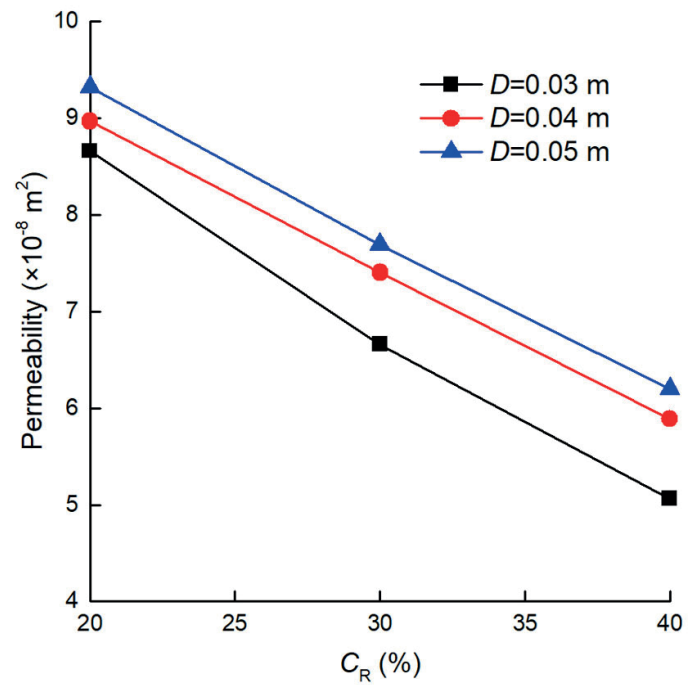

Fig. 10 The influence of rock content and rock size on the permeability of SRMs with bolck-shaped rocks

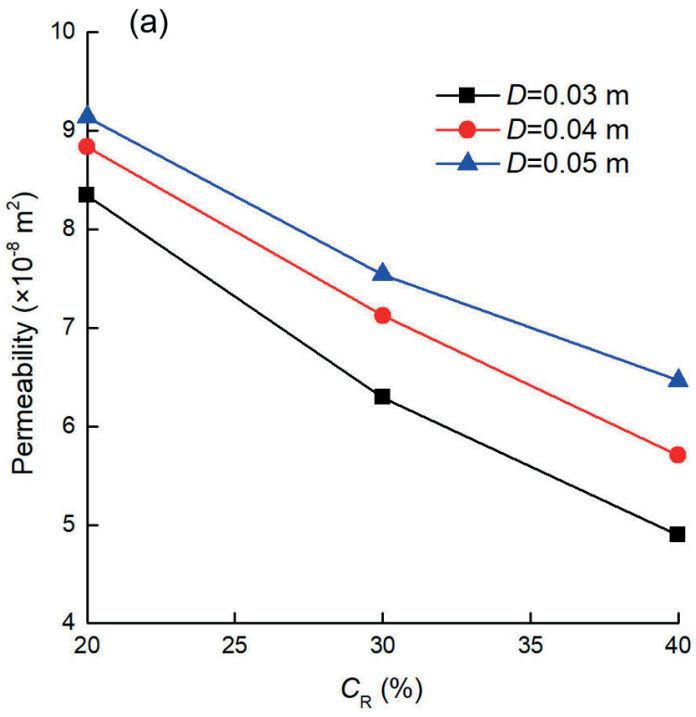

(b)

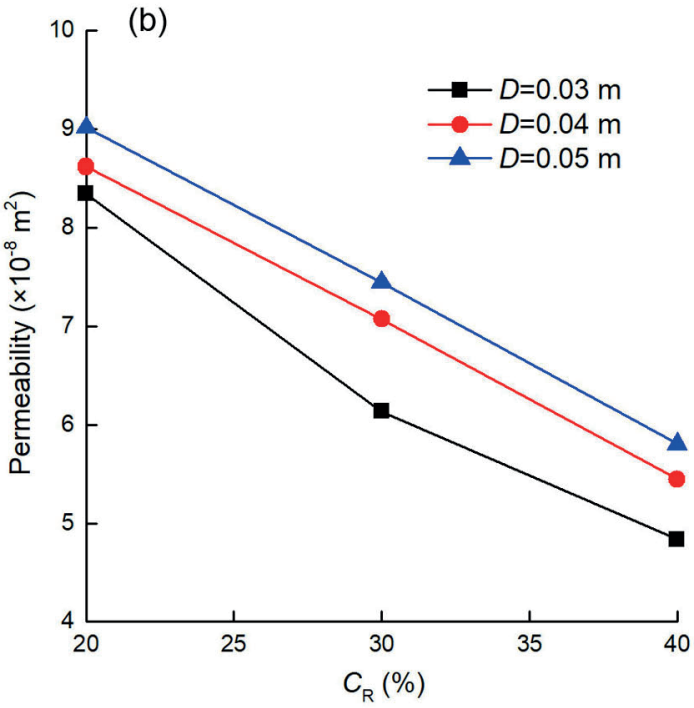

(c)

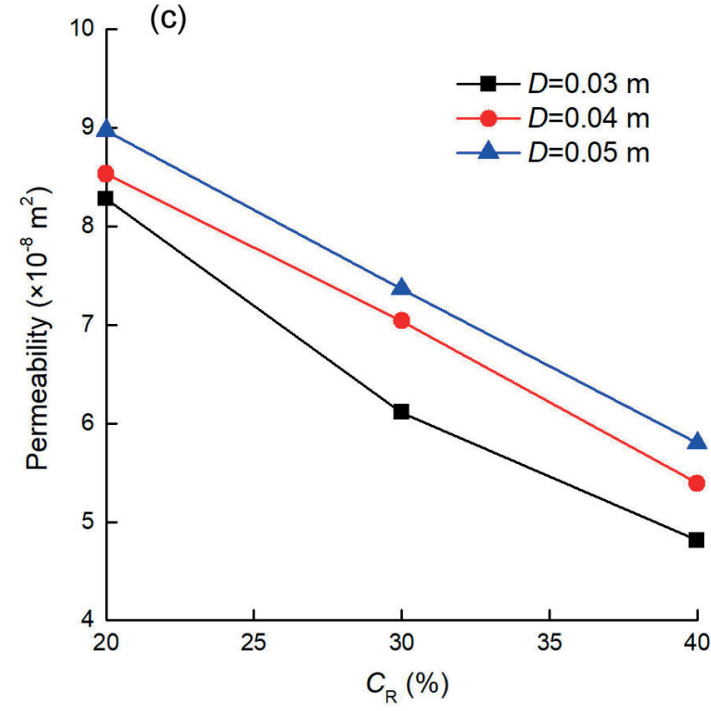

Fig. 11 The influence of rock content and rock size on the permeability of SRMs with bar-shaped rocks. (a) $\theta=0^{\circ}$, (b) $\theta=$ random, (c) $\theta=90^{\circ}$ 

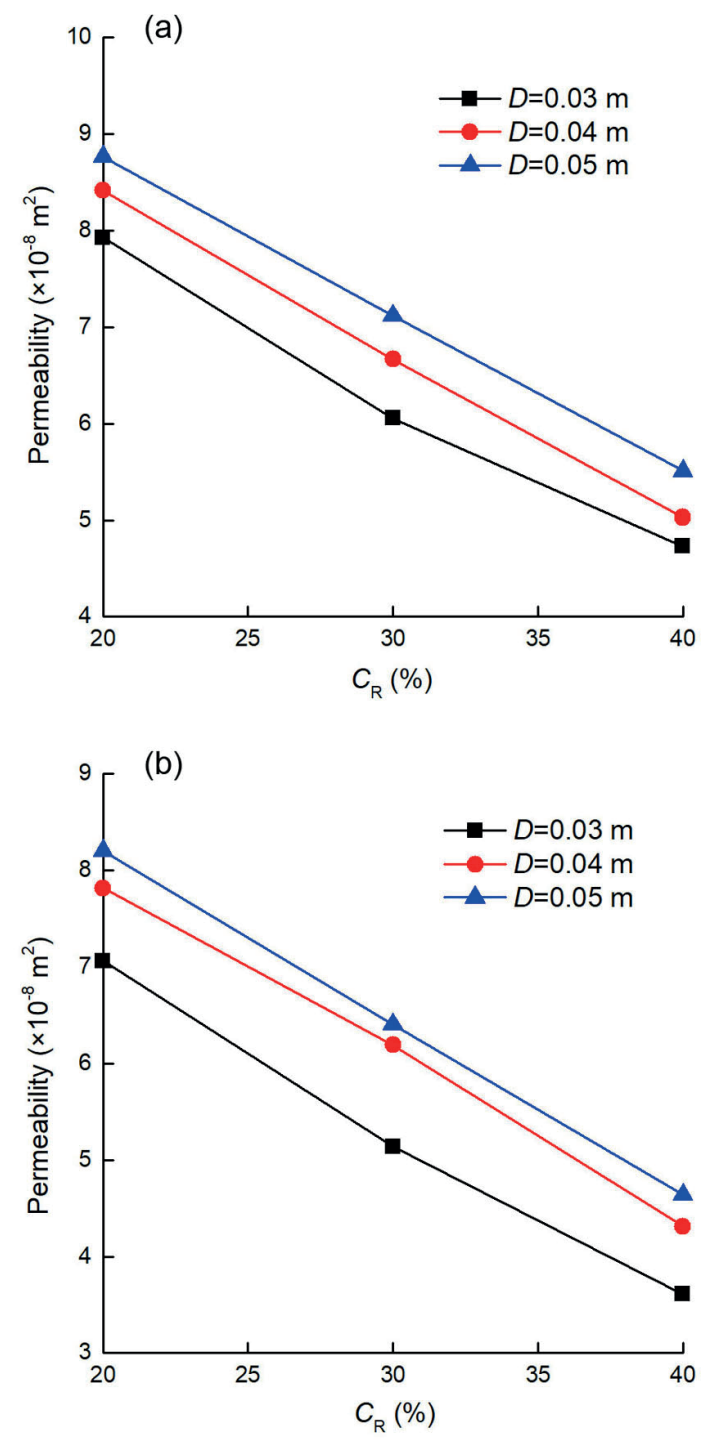

(c)

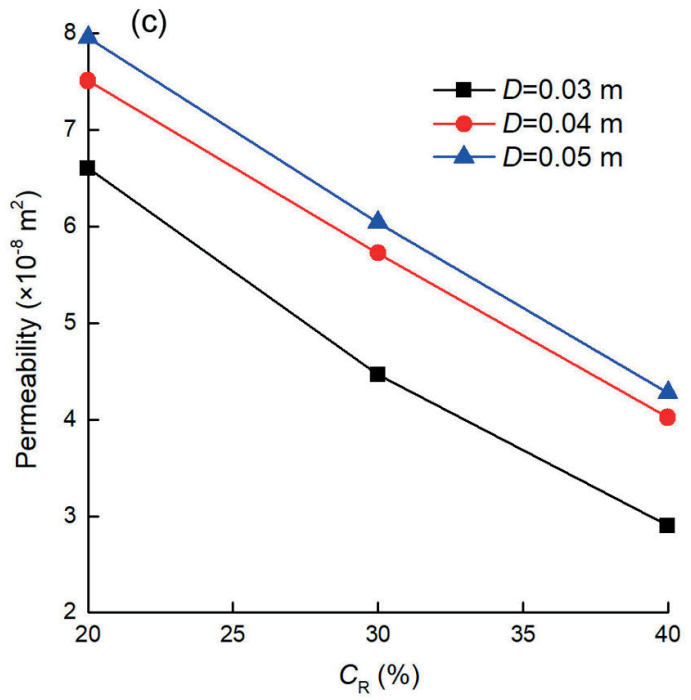

Fig. 12 The influence of rock content and rock size on the permeability of SRMs with slab-shaped rocks. (a) $\theta=0^{\circ}$, (b) $\theta=$ random, (c) $\theta=90^{\circ}$
The porosity of soil matrix in SRMs are kept to be 0.40 in this paper. Therefore, as the rock content increases, the porosity of SRMs decreases, so the permeability gradually decreases. For example, regarding the SRMs with block-shaped rocks of sizes $0.04 \mathrm{~m}$, as the rock content increases from $20 \%$ to $40 \%$, the porosity decreases from 0.3221 to 0.2566 , and thus the permeability drops from $8.968 \times 10^{-8} \mathrm{~m}^{2}$ to $5.889 \times 10^{-8} \mathrm{~m}^{2}$. Previous laboratory tests and numerical simulations $[7,8,10]$ also reported similar conclusions.

However, it should be noted that some other previous studies concluded that the permeability of SRMs increases with rock content, which is contrary to the conclusion of this work. This may be due to the fact that it is the compaction degree of SRMs rather than the porosity of soil matrix that did not change in their experiments for SRMS with different rock contents. The distinct effects of rock content on permeability of SRMs under different test conditions will be investigated in another paper.

Chen et al. [10] simulated the seepage of SRMs with two-dimensional numerical manifold method and concluded that the influence of rock size on the permeability of SRMs can be neglected. However, according to the results of three-dimensional LB simulation presented in this paper, the rock size does have some influence on the permeability of SRMs. It is known that the specific surface of a solid particle decreases with its size. Therefore, when the other conditions remain unchanged, the specific surface of SRMs decreases with the increase of rock size, so the permeability gradually increases.

\subsection{Influence of rock shape}

Fig. 13 shows the influence of rock shape on the permeability of SRMs. It can be seen that the rock shape has some influence on the permeability of SRMs. If $\theta=$ random, the permeability of SRMs with bar-shaped rocks is smaller than that of SRMs with block-shaped rocks, but larger than that of SRMs with slab-shaped rocks.

This influence of rock shape on the permeability of SRMs can also be attributed to the effect of specific surface. The specific surfaces are calculated to be $6 / D$ for the block-shaped rock, 8.6/D for the bar-shaped rock and 10/D for the slab-shaped rock, respectively. Therefore, when the other conditions remain unchanged, the specific surface of SRMs increases causing the permeability to decrease as the rock shape changes from block, bar to slab. 

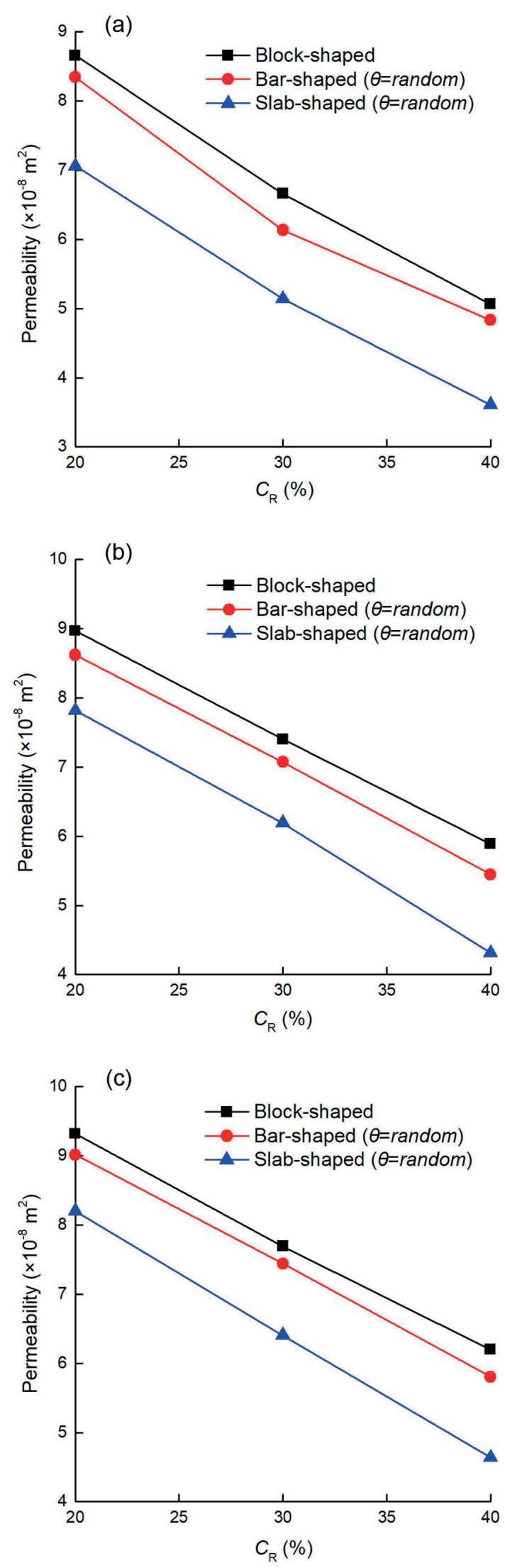

Fig. 13 The influence of rock shape on the permeability of SRMs; (a) $D=0.03 \mathrm{~m}$, (b) $D=0.04 \mathrm{~m}$, (c) $D=0.05 \mathrm{~m}$

\subsection{Influence of rock orientation}

As can be seen from Fig. 14, the rock orientation has a certain influence on the permeability of SRMs, and the amount of variation changes with the rock shape. To be specific, when the rocks are bar-shaped, the permeability is slightly decreased as the major axes of these rocks change from parallel $\left(\theta=0^{\circ}\right)$ to perpendicular $\left(\theta=90^{\circ}\right)$ with respect to the direction of main flow; when the rocks are slab-shaped, the permeability decreases more significantly as the slab planes of these rocks change from parallel $\left(\theta=0^{\circ}\right)$ to perpendicular $\left(\theta=90^{\circ}\right)$ with respect to the direction of main flow.

$\mathrm{Xu}$ and Wang [8] and Chen et al. [10] studied the influence of rock orientation on the permeability of SRMs with elliptical rocks, and concluded that the permeability gradually decreases as the angles increase between the major axes of rocks and the direction of main flow. The results of LB simulation in this study also conform to this conclusion.

When the rock orientation is varied individually, the porosity and specific surface of SRMs are both kept the same, but the tortuosity of the flow paths is different. Fig. 15 shows the pore-scale seepage field of the intermediate longitudinal sections in the SRMs $\left(C_{\mathrm{R}}=30 \%\right.$, $D=0.04 \mathrm{~m}$ and slab-shaped). As can be seen, when $\theta=90^{\circ}$, the area occupied by the rocks is relatively larger in the cross-section, so the flow paths are more curved, and thus the permeability is decreased. The results of two-dimensional LB simulations conducted by Nabovati and Sousa [14] also indicate that the tortuosity is larger of the porous medium with rectangular solid particles of high aspect ratios (corresponding to $\theta=90^{\circ}$ ). Compared with the bar-shaped rock, the slab-shaped rock can occupy a larger proportion of the cross section when turning perpendicular to the flow direction, which makes its blocking effect on the seepage paths more significant and its influence on permeability more obvious.

\section{Conclusions}

This research represents the first attempt of using the three-dimensional LBM to simulate the seepage in SRMs from the pore scale. The influences of rock content, rock size, rock shape and rock orientation are comprehensively investigated on the permeability of SRMs.

Based on the results of present study, the following conclusions can be made:

1. Three-dimensional pore-structural models of SRMs with different rock contents, rock sizes, rock shapes and rock orientations can be constructed with the 

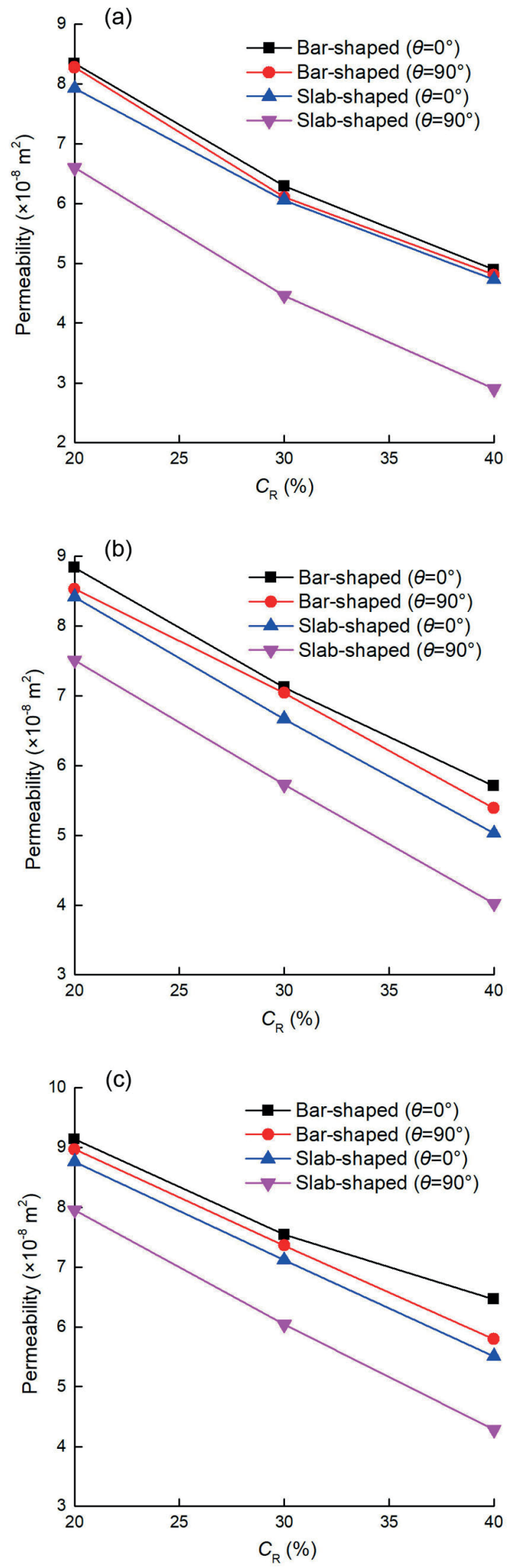

Fig. 14 The influence of rock orientation on the permeability of SRMs; (a) $D=0.03 \mathrm{~m}$, (b) $D=0.04 \mathrm{~m}$, (c) $D=0.05 \mathrm{~m}$ (a)

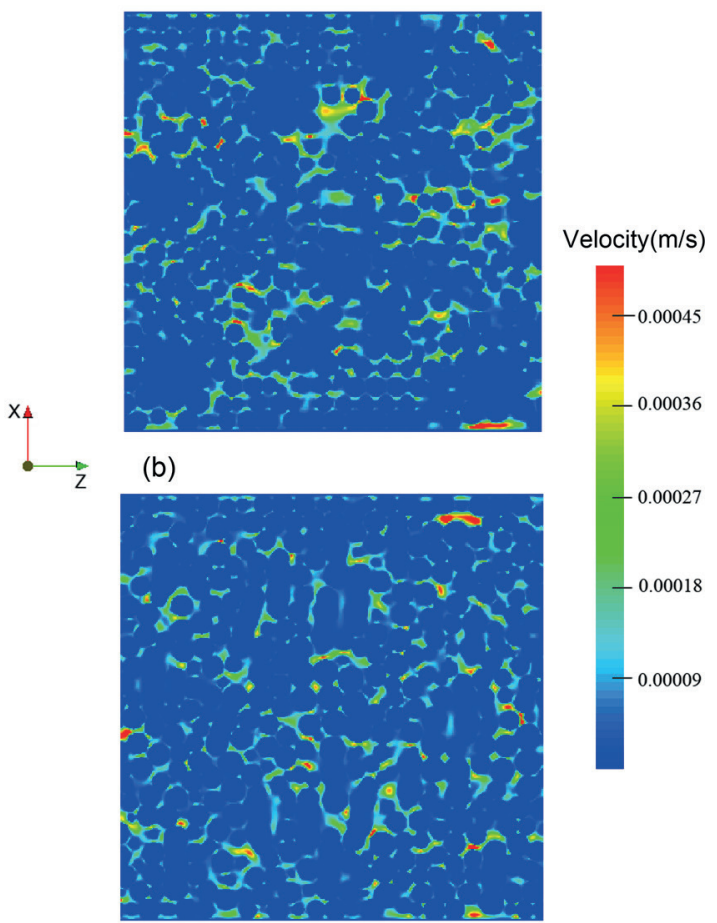

Fig. 15 Velocity contour plots of the intermediate longitudinal section in the SRMs $\left(C_{\mathrm{R}}=30 \%, D=0.04 \mathrm{~m}\right.$ and slab-shaped); (a) $\theta=0^{\circ}$, (b) $\theta=90^{\circ}$

discrete element method, and they can be voxelized with the proposed virtual slicing technique for further LB simulation of seepage.

2. The permeability of SRMs decreases with rock content while increases with rock size.

3. The permeability of SRMs with bar-shaped rocks is smaller than that of SRMs with block-shaped rocks, but larger than that of SRMs with slab-shaped rocks.

4. The rock orientation has a certain influence on the permeability of SRMs, and this influence relates to the rock shape.

Additionally, the DEM modeling method and the LB simulation method presented in this paper can be used to further study the influences of other factors, such as the rock gradation and confining pressure, on the permeability of SRMs.

\section{Acknowledgement}

The project presented in this article is supported by the National Natural Science Foundation of China (no. 41272342) and Natural Science Foundation of Hubei Province (no. 2019CFB199). 


\section{References}

[1] Xu, W., Yue, Z., Hu, R. "Study on the mesostructure and mesomechanical characteristics of the soil-rock mixture using digital image processing based finite element method", International Journal of Rock Mechanics and Mining Sciences, 45(5), pp. 749-762, 2008. https://doi.org/10.1016/j.ijrmms.2007.09.003

[2] Medley, E. W. "Orderly Characterization of Chaotic Franciscan Melanges", Felsbau, 19(4), pp. 20-33, 2001. [online] Availableat: https://www.bimrocks.geoengineer.org/files/MedleyFelsbau 2001.pdf

[3] Wang, Y., Li, J., Jiang, Q., Huang, Y., Li, X. "Study on Spatial Variation of Shear Mechanical Properties of Soil-rock Mixture", Periodica Polytechnica Civil Engineering, 63(4), pp. 1080-1091, 2019.

\section{https://doi.org/10.3311/PPci.14769}

[4] Graziani, A., Rossini, C., Rotonda, T. "Characterization and DEM Modeling of Shear Zones at a Large Dam Foundation", International Journal of Geomechanics, 12(6), pp. 648-664, 2012. https://doi.org/10.1061/(ASCE)GM.1943-5622.0000220

[5] Gao, Q., Liu, Z. H., Li, X. "露天坑回填土石混合体的渗流特性 及颗粒元数值分析" (Permeability characteristics of rock and soil aggregate of backfilling open-pit and particle element numerical analysis), Chinese Journal of Rock Mechanics and Engineering, 28(11), pp. 2342-2348, 2009. (in Chinese) https://doi.org/10.3321/j.issn:1000-6915.2009.11.025

[6] Zhou, Z., Yang, H., Wang, X., Liu, B. "Model Development and Experimental Verification for Permeability Coefficient of Soil-Rock Mixture", International Journal of Geomechanics, 17(4), Article No. 04016106, 2017. https://doi.org/10.1061/(ASCE)GM.1943-5622.0000768

[7] Wang, Y., Li, X., Zheng, B., Li, S. D., Duan, Y. T. "A laboratory study of the effect of confining pressure on permeable property in soil-rock mixture", Environmental Earth Sciences, 75(4), Article No. 284, 2016. https://doi.org/10.1007/s12665-015-5193-X

[8] Xu, W. J., Wang, Y. G. "土石混合体细观结构渗流数值试验研究" (Meso-structural permeability of S-RM based on numerical tests), Chinese Journal of Geotechnical Engineering, 32(4), pp. 542-550, 2010. (in Chinese) [online] Available at: http://manu31.magtech. com.cn/Jwk_ytgcxb/CN/article/downloadArticleFile.do?attachType $=$ PDF\&id=12440\&feeFlag=true [Accessed: 15 April 2010]

[9] Yan, L., Meng, Q., Xu, W., Wang, H., Zhang, Q., Zhang, J., Wang, R. "A numerical method for analyzing the permeability of heterogeneous geomaterials based on digital image processing", Journal of Zhejiang University-SCIENCE A, 18(2), pp. 124-137, 2017. https://doi.org/10.1631/jzus.A1500335

[10] Chen, T., Yang, Y., Zheng, H., Wu, Z. "Numerical determination of the effective permeability coefficient of soil-rock mixtures using the numerical manifold method", International Journal for Numerical and Analytical Methods in Geomechanics, 43(1), pp. 381-414, 2019. https://doi.org/10.1002/nag.2868

[11] Chen, S., Doolen, G. D. "Lattice Boltzmann method for fluid flows", Annual Review of Fluid Mechanics, 30, pp. 329-364, 1998. https://doi.org/10.1146/annurev.fluid.30.1.329
[12] Succi, S., Foti, E., Higuera, F. "Three-Dimensional Flows in Complex Geometries with the Lattice Boltzmann Method", Europhysics Letters, 10(5), pp. 433-438, 1989. https://doi.org/10.1209/0295-5075/10/5/008

[13] Pan, C., Hilpert, M., Miller, C. T. "Pore-scale modeling of saturated permeabilities in random sphere packings", Physical Review E, 64(6), Article No. 066702, 2001.

https://doi.org/10.1103/PhysRevE.64.066702

[14] Nabovati, A., Sousa, A. C. M. "Fluid Flow Simulation in Random Porous Media at Pore Level Using Lattice Boltzmann Method", In: Zhuang, F. G., Li, J. C. (eds.) New Trends in Fluid Mechanics Research, Springer, Berlin, Heidelberg, 2007, pp. 518-521. https://doi.org/10.1007/978-3-540-75995-9_172

[15] Ghassemi, A., Pak, A. "Pore scale study of permeability and tortuosity for flow through particulate media using Lattice Boltzmann method", International Journal for Numerical and Analytical Methods in Geomechanics, 35(8), pp. 886-901, 2011. https://doi.org/10.1002/nag.932

[16] Han, Y., Cundall, P. A. "Lattice Boltzmann modeling of pore-scale fluid flow through idealized porous media", International Journal for Numerical Methods in Fluids, 67(11), pp. 1720-1734, 2011. https://doi.org/10.1002/fld.2443

[17] Takbiri Borujeni, A., Lane, N. M., Thompson, K., Tyagi, M. "Effects of image resolution and numerical resolution on computed permeability of consolidated packing using LB and FEM porescale simulations", Computers and Fluids, 88, pp. 753-763, 2013. https://doi.org/10.1016/j.compfluid.2013.05.019

[18] Wang, M., Feng, Y. T., Wang, C. Y. "Numerical investigation of initiation and propagation of hydraulic fracture using the coupled Bonded Particle-Lattice Boltzmann Method", Computers and Structures, 181, pp. 32-40, 2017. https://doi.org/10.1016/j.compstruc.2016.02.014

[19] Wang, M., Feng, Y. T., Pande, G. N., Zhao, T. T. "A coupled 3-dimensional bonded discrete element and lattice Boltzmann method for fluid-solid coupling in cohesive geomaterials", International Journal for Numerical and Analytical Methods in Geomechanics, 42(12), pp. 1405-1424, 2018. https://doi.org/10.1002/nag.2799

[20] Wang, M., Feng, Y. T., Owen, D. R. J., Qu, T. M. "A novel algorithm of immersed moving boundary scheme for fluid-particle interactions in DEM-LBM", Computer Methods in Applied Mechanics and Engineering, 346, pp. 109-125, 2019. https://doi.org/10.1016/j.cma.2018.12.001

[21] Cousins, T. A., Ghanbarian, B., Daigle, H. "Three-Dimensional Lattice Boltzmann Simulations of Single-Phase Permeability in Random Fractal Porous Media with Rough Pore-Solid Interface", Transport in Porous Media, 122, pp. 527-546, 2018. https://doi.org/10.1007/s11242-017-0938-5

[22] Xu, W.-J., Wang, S., Zhang, H.-Y., Zhang, Z.-L. "Discrete element modelling of a soil-rock mixture used in an embankment dam", International Journal of Rock Mechanics and Mining Sciences, 86, pp. 141-156, 2016. https://doi.org/10.1016/j.ijrmms.2016.04.004 
[23] Jin, L., Zeng, Y., Xia, L., Ye, Y. "Experimental and Numerical Investigation of Mechanical Behaviors of Cemented Soil-Rock Mixture", Geotechnical and Geological Engineering, 35, pp. 337354, 2017. https://doi.org/10.1007/s10706-016-0109-4

[24] Cundall, P. A., Strack, O. D. L. "Discussion: A discrete numerical model for granular assemblies", Géotechnique, 30(3), pp. 331-336, 1980. https://doi.org/10.1680/geot.1980.30.3.331

[25] Cho, N., Martin, C. D., Sego, D. C. "A clumped particle model for rock", International Journal of Rock Mechanics and Mining Sciences, 44(7), pp. 997-1010, 2007. https://doi.org/10.1016/j.ijrmms.2007.02.002

[26] Bhatnagar, P. L., Gross, E. P., Krook, M. "A Model for Collision Processes in Gases. I. Small Amplitude Processes in Charged and Neutral One-Component Systems", Physical Review, 94(3), pp. 511-525, 1954.

https://doi.org/10.1103/PhysRev.94.511

[27] d'Humières, D., Ginzburg, I., Krafczyk, M., Lallemand, P., Luo, L.-S. "Multiple-relaxation-time lattice Boltzmann models in three dimensions", Philosophical Transactions of The Royal Society A, Mathematical Physical and Engineering Sciences, 360, pp. 437451, 2002.

https://doi.org/10.1098/rsta.2001.0955

[28] Pan, C., Luo, L.-S., Miller, C. T. "An evaluation of lattice Boltzmann schemes for porous medium flow simulation", Computers and Fluids, 35(8-9), pp. 898-909, 2006.

https://doi.org/10.1016/j.compfluid.2005.03.008
[29] Jin, L., Zeng, Y.-W., Ye, Y., Li, J.-J. "不规则颗粒及其集合体三 维离散元建模方法的改进" (Improving three-dimensional DEM modeling methods for irregularly shaped particles and their assembly), Chinese Journal of Geotechnical Engineering, 39(7), pp. 12731281, 2017. (in Chinese) https://doi.org/10.11779/CJGE201707014

[30] Zou, Q., He, X. "On pressure and velocity boundary conditions for the lattice Boltzmann BGK model", Physics of Fluids, 9(6), pp. 1591-1598, 1997. https://doi.org/10.1063/1.869307

[31] University of Geneva "Palabos, (v2.0r0)", [computer program] Available at: https://palabos.unige.ch

[32] Koponen, A., Kataja, M., Timonen, J. "Permeability and effective porosity of porous media", Physical Review E, 56(3), pp. 33193325, 1997. https://doi.org/10.1103/PhysRevE.56.3319

[33] Chapuis, R. P. "Predicting the saturated hydraulic conductivity of sand and gravel using effective diameter and void ratio", Canadian Geotechnical Journal, 41(5), pp. 787-795, 2004. https://doi.org/10.1139/t04-022

[34] Feng, Y. T., Han, K., Owen, D. R. J. "Coupled lattice Boltzmann method and discrete element modeling of particle transport in turbulent fluid flows: Computational issues", International Journal for Numerical Methods in Engineering, 72(9), pp. 1111-1134, 2007. https://doi.org/10.1002/nme.2114 